\title{
Presbycusis: A Critical Issue in Our Community
}

\author{
Ming Zhang ${ }^{1,2,3}$, Nahla Gomaa ${ }^{3,4}$, Allan $\mathrm{Ho}^{3,4}$ \\ ${ }^{1}$ Department of Speech Pathology and Audiology, University of Alberta, Edmonton, Canada \\ ${ }^{2}$ Department of Audiology, Glenrose Rehabilitation Hospital, Edmonton, Canada \\ ${ }^{3}$ Department of Otolaryngology and Head \& Neck Surgery, University of Alberta, Edmonton, Canada \\ ${ }^{4}$ Department of Otolaryngology and Head \& Neck surgery, Grey Nuns Hospital, Edmonton, Canada \\ Email: ming.zhang@ualberta.ca
}

Received April 23, 2013; revised May 24, 2013; accepted June 15, 2013

Copyright (C) 2013 Ming Zhang et al. This is an open access article distributed under the Creative Commons Attribution License, which permits unrestricted use, distribution, and reproduction in any medium, provided the original work is properly cited.

\begin{abstract}
Hearing is an important sensation to the elderly as it promotes their quality of life and maintains their safety and wellness. For example, healthy hearing lets the elderly catch alarm sounds, stay alert to danger whilst asleep, listen in the dark, detect sounds from behind, communicate efficiently with other people, and maintain links to the world via telephone and radio, especially after retirement. However, age-related hearing loss, i.e., presbycusis, seems to become a growing problem in our community. The purpose of this study is to demonstrate whether presbycusis is a critical issue in our community. To achieve this purpose, the data in the literature as well as in the websites sponsored by hearing-related professional associations and sponsored by related government's departments have been searched and reviewed. The data resulted from the review show a high prevalence of presbycusis, an ever-growing senior population, an incredible increase in hearing impairment and presbycusis population in the next two decades, a rank as high as at the third place for the prevalence of presbycusis among chronic health conditions in elderly resident facilities, and an alarmingly negative effect of presbycusis on mental health, social life, speech perception and hearing-related areas in the brain. These findings demonstrate that hearing loss in the elderly is a critical issue in our community. The etiology, clinical significance, management of presbycusis, prevention, and access of presbycusis population to assistive devices are also overviewed and discussed.
\end{abstract}

Keywords: Presbycusis; Age-Related Hearing Loss; Aging; Prevalence

\section{Introduction}

Hearing is one of the most important senses to the elderly. Being able to hear allows the elderly to catch alarming sounds, to stay alert during sleep, to listen in the dark, and to detect sound from behind. Additionally, the incidence of various age-related disabilities is known to be high in the elderly group. Hence, they heavily rely on special senses such as hearing to compensate for other age-related disabilities [1]. Moreover, the elderly also need to rely on good hearing to communicate efficiently with family members and maintain links to the world via telephone and radio after retirement.

In contrast, the inability to hear due to hearing loss would substantially affect all these functions above mentioned, and therefore the quality of life in the elderly would be substantially reduced. For example, hearing loss can be an important factor that contributes to isolation, depression, and possibly dementia in the elderly [2-4].
As early as 1993, Schuknecht, a pioneer otologist in our field, attended this presbycusis issue in his report on Ann Otol Rhinol Laryngol [5]. As late as current days, in our daily activities, presbycusis still very often appears as a major issue. Therefore, as an otology-audiology team affiliated to four facilities, we started a series of studies on presbycusis recently. We found that solid reports to extensively demonstrate presbycusis as a critical issue is rare. Therefore, our first study in this series was to demonstrate whether presbycusis would be a critical issue to our community, also being the hypothesis of this study.

There are a number of aspects that are associated with this hypothesis such as prevalence of presbycusis [6], growth of senior population segment [7-9], projection of prevalence of presbycusis, ranking of prevalence of presbycusis among chronic health conditions in elderly residents [9-13], negative effects of presbycusis on subject's quality of life (e.g., mental health, social life, and speech perception) [14-16], and clinical significance (e.g., management and prevention of the presbycusis). 
By studying these issues, the hypothesis can be tested or supported. For example, prevalence as an epidemiological term is the proportion of a population found to have a condition. It can be expressed as a percentage at a specific point in time [17]. A range of prevalence of presbycusis can be between $25 \%$ in those at 70 - 74 years old, $50 \%$ by age 85 , and $>80 \%$ at 85 years old or above [18-20]. The high prevalence in the elderly is one aspect to support that the presbycusis is a critical issue in our community.

This study can be significant. For example, it can be used as a solid reference and foundation to support initiation of further studies on presbycusis and to support research funding application for the presbycusis study. In addition, a report from this study is potentially of useful information to the clinics and patients in daily clinical activities.

\section{Methods}

\subsection{Review Approach}

We have undergone an extended review process. This extended review process is based on the recent articles on review process methods [21,22]. An extended review for this study is to demonstrate that the presbycusis is a critical issue. Such demonstration requires high quality convincing data rather than large quantity of the data. Study on this critical issue of presbycusis is still at its early stage and far from well-developed stage. Therefore, the number of reports directly studying this topic is limited. For example, the number of people with presycusis and hearing loss is usually indirectly estimated. An extended review process is needed to cover those most important articles in the literature instead of all articles which are trivial. After we started this study, we became understanding that an extended review process is needed as not one review process can fit all studies [21,22].

\subsection{Review Stages}

Our extended review process covered five stages. The first stage was a literature search into three major databases related to several main domains (speech pathology, audiology, and health care). The databases include the Medline/PubMed, Cochrane database, and Google scholar. A variety of search strings were used; and they are relatively broad in concept in an attempt to include and explore as many related reports as possible. The strings containing presbycusis or age-related hearing loss were used. This first search stage yielded about 375 results.

The second stage was to perform a critical appraisal for individual papers by reading the abstracts to decide which of them can be excluded as majority of the results were irrelevant. The exclusion criteria included animal studies, case studies with limited scope, incidence studies with small sample size, genetic studies on specific iso- lated molecules, outdated studies, stem cell studies, hair cell regeneration studies, some anatomical and morphological studies such as increase of hairs in the external ears, cell and molecular studies without direct clinical significance, studies emphasizing other topics such as tinnitus, treatment studies using a specific remedy with uncertainty. After exclusion of those irrelevant results, about 70 results were identified as relevant to this study.

The third stage was through a general search into Google search engine using the same search strings as described above. Some with relevant information were identified. They are listed with http in the references.

The fourth stage was through a search into multiple government-related websites as well as into multiple formal professional association-related websites. These websites, listed alphabetically, include the American Academy of Audiology, the American Speech-Language-Hearing Association, the Canadian Association of SpeechLanguage Pathologists and Audiologists, the National Institutes of Health, the National Science Foundation, the United Nations, United Nations Population Fund (UNFPA), and the World Health Organization. The aim of this stage was to collect data related to the logistic aspects related to presbycusis.

Finally in the fifth stage, various libraries and publishers were contacted to obtain some relevant articles we had identified.

\section{Findings and Discussions}

Based on our hypothesis, our findings are focused to demonstrate one fact that presbycusis is a critical issue in our community. The data we have based on are extended into following several aspects with the detail further presented and discussed in eight (8) subsections:

3.1. "Presbycusis: Etiology";

3.2. "Prevalence of Hearing Loss: Increase with Age";

3.3. "Seniors: The Fastest Growing Segment of Society";

3.4. "Projection: Potential of Upcoming Significant Increase in Prevalence of Presbycusis";

3.5. "Hearing Impairment: Ranking the Third Place in Prevalence among All Chronic Health Conditions in Elderly Residents";

3.6. "Hearing Loss in Elderly Neuroscience (I): A Negative Factor on Mental Health and Social life";

3.7. "Hearing Loss in Elderly Neuroscience (II): A Negative Effect on Speech Perception and Hearing-Related Areas in the Brain"; and

3.8. "Clinical Significance: Challenge in Management and Prevention of the Presbycusis".

\subsection{Presbycusis: Etiology}

Presbycusis is a Greek term means old and hearing [23]. 
Presbycusis is very common in the elderly. In a number of studies addressing factors associated with hearing impairment (HI), the age distribution of hearing impairment was substantially constant with the highest prevalence rates observed among older adults $[4,6,18]$.

The presbycusis have been divided based on the etiology into three subtypes. Sensory presbycusis caused by permanent damage and loss of hair cells in the basal cochlea and is often associated with noise exposure. Strial presbycusis, however, results from declining metabolic function of the cochlea. The third type-neural presbycusis - was attributed to degeneration of the auditory nerve $[5,24]$. However, gene-related hereditary susceptibility has also been shown to play an importance role in the progress of presbycusis $[25,26]$.

The aging process in the ear can also occur in the external ear and middle ear. However, the dominant cause occurs in the cochlea which is the hearing portion of the inner ear [5], and is due to a loss of hair cells in the cochlea [27]. The hair cells are critical to normal hearing function as they convert sound into nerve activities, and yet they are very vulnerable [28]. In mammals like humans, the hair cells live only once; once they are lost, the loss is permanent. In addition, unlike the eye which has an eye-lid, the ear does not have ear-lid, and they have to work day and night. Therefore, everyone, no matter how healthy, will develop presbycusis sooner or later due to the constant loss of a few hair cells every a while.

The age-related changes in the central nervous system can also be associated with presbycusis, which is addressed in the late Sections 3.6 and 3.7 (Hearing Loss in Elderly Neuroscience).

\subsection{Prevalence of Hearing Loss: Increase with Age}

In the North America, around $10 \%$ of the population (e.g., more than 30,000,000 individuals in the USA and more than 3,000,000 individuals in Canada) suffer from hearing loss with the highest prevalence rates among aged population such as those aged 65 or above [6]. Aging is one of the most important causes of hearing loss [29]. Elderly population relies on special senses to compensate for other age-related disabilities and also for slowed reaction time. In addition, age-related decline in the ability of mental concentration and memory also contributes to their difficulty in understanding speech [1]. Whether corrected or uncorrected, hearing impairment is highly prevalent in later life regardless of other socio-demographic factors such as marital status, education, and income [30].

Prevalence of hearing loss increases with age although the results may vary among various reports in literature. For example, increase in prevalence of hearing loss with age has occurred in the USA. Statistics reported in the
American Speech-Language-Hearing Association shows that hearing loss is the third most prevalent chronic condition in older Americans. Hearing loss is the number one communicative disorder in the elderly population, e.g., $25 \%$ to $40 \%$ of the population aged 65 years or older is hearing impaired [18,31]. Willott et al. reported that hearing loss starts as early as the third or fourth decade of life [32]. For example, between $25 \%$ and $48 \%$ of adults aged 75 - 79 years have some degree of hearing loss measured with audiometry.

Equivalently, in agreement with national figures in the USA, the Canadian Association of Speech-Language Pathologists and Audiologists confirms in its fact sheet that $20 \%$ of adults over $65,40 \%$ over 75 and $80 \%$ of nursing home residents have a significant hearing problem [33].

The older the population is, the higher the prevalence will be. For example, a range of prevalence between 25\% of those at 70 - 74 years old and $50 \%$ by age 85 was reported $[19,20]$, and also a range between $40 \%$ - $66 \%$ of those at $>75$ years old and $>80 \%$ at 85 years old or above [18].

Certain factors were considered in some studies which may be associated with hearing impairment, e.g., gender, age, etc. For example, across the gender, the age distribution of hearing impairment was substantially constant, i.e., the highest prevalence rates were among both male and female older adults (aged 65 and older) [6,18,30]. However, when relating the prevalence of hearing loss to the level of education, it was reported that the prevalence is higher among less educated groups [13,34], a finding that was probably attributed to limited access to health services among people with low levels of education, or exposure to ototoxic factors such as noise or medications among this group of population.

By reviewing the data collated above, variation in prevalence reported by different investigators can be observed. The variation in results may reflect the difference in the definition of the age of "elderly". The lack of a maximum age in the age group in some reports may also be considered as a contributor to the variation in data to a certain extent. The differences in the methods that each study adopted for sampling the population into groups at different age range as well as the differences inherent in geographically distinct people groups may be regarded as the major contributing factors. For example, difference in the estimates of hearing loss depends on the choice of criterion for determining whether a hearing loss may exist. Estimates based on audiometric measures of impairment may be higher than estimates based on subjective and qualitative indices of handicap [12]. Estimates based on audiometric measures may be lower than actual figures because they often do not reflect conditions of central auditory processing. 
However, although different, the data among the multiple studies above are not contradictory to each other if these data are overlapped together across the ages in terms of rate which is rising in presbycusis population. This rising trend can be clearly visualized in Figure 1 plotted based on the data combined from the abovementioned reports in the literature $[3,6,18,30,32]$.

\subsection{Seniors: The Fastest Growing Segment in Our Society}

According to a statistic data [33], seniors represent the fastest growing segment of the population. This segment is expected to make up $1 / 4$ of the population (i.e., 25\%) by 2041. The senior population has increased over years based on the available data and prediction: $10.6 \%$ in $1991,12 \%$ in $1993,14.5 \%$ by $2011,21.8 \%$ in 2031 , and $25 \%$ in 2041 [7-9]. The number $21.8 \%$ in 2031 is more than double than that in 1991 (10.6\%). The fast growing trend can clearly be visualized if data are displayed in a plot format (Figure 2).

This ever-increasing percentage of seniors in a total population is an additional factor further contributing to the increase in the number of hearing loss in the elderly. With an increasing prevalence of hearing impairment with age, if senior population rapidly expands with time year by year, the total number of elderly people with hearing loss will increase at an even faster rate among whole population. For example, in the elderly above age 65 years or older, the prevalence of hearing impaired can be $40 \%[18,31]$, while in the adult under age of 65 , the prevalence of $20 \%$ can be reasonably assumed. Then, the ratio can be doubled by comparison $(40 \% / 20 \%=200 \%)$. Even more severe is that the number of hearing loss will be further doubled (i.e., $400 \%=200 \% \times(21.8 \% / 10.6 \%)$ if the population of the people at age 65 is doubled (21.8\% in 2031 over $10.6 \%$ in 1991).

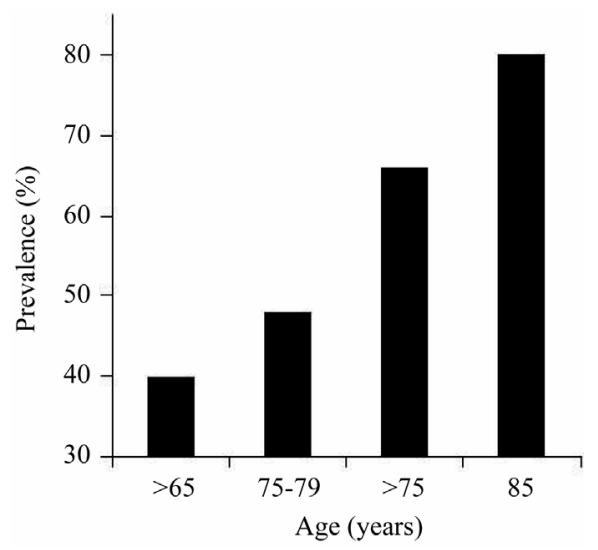

Figure 1. Prevalence of hearing loss as a function of age. The prevalence of hearing loss is presented as percentage of the population in each age group. The prevalence of hearing loss increases with age.

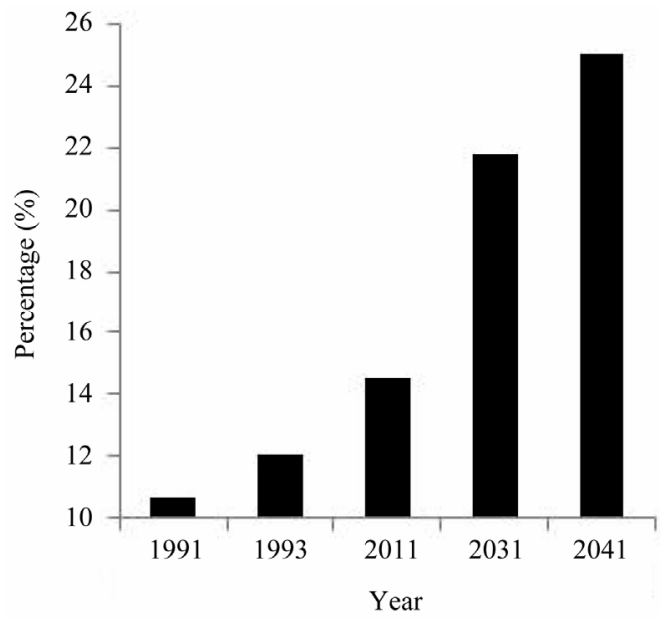

Figure 2. Change of senior population size as a function of year. The change of senior population size is presented as percentage of the whole population of all age groups in each year. The size of the senior population increases with year.

Even if the percentage $10.6 \%$ in 1991 does not increase to $21.8 \%$ in 2031 for senior age group, provided that the total population including all age groups increases with time, the absolute number of seniors will increase accordingly with other age groups as well. Considering that the population over all age groups has been continuously increasing in the past decades. There is no sign that this trend will be halted.

Based on these three factors (the increase in hearing loss with age, the increase in percentage of seniors in the whole population, and the increase in whole population in all age groups), it is evident that the absolute number of the elderly who suffer from hearing loss will substantially increase in the foreseeable future.

\subsection{Projection: Potential of Upcoming Significant Increase in Prevalence of Presbycusis}

Hearing loss has become an important issue as viewed by the World Health Organization (www.who.int). They have done a series of studies on the number of people who suffer from hearing impairment. They found that hearing impairment affected more than 120 million people worldwide in 1999, 278 million in 2005, and 642 million in 2011.

It will be interesting to estimate how many people will be affected by 2017, by 2025, and even by 2031. For example, the senior population is estimated to increase up to $21.8 \%$ by 2031 as shown in Figure 3 [7-9]. We propose an estimated projection that hearing impairment will affect 1248 million people in 2017, affect 2568 million people in 2025, and 5136 million in 2031. We believe that these three numbers are relatively reasonable due to following two reasons. First, by looking at the 


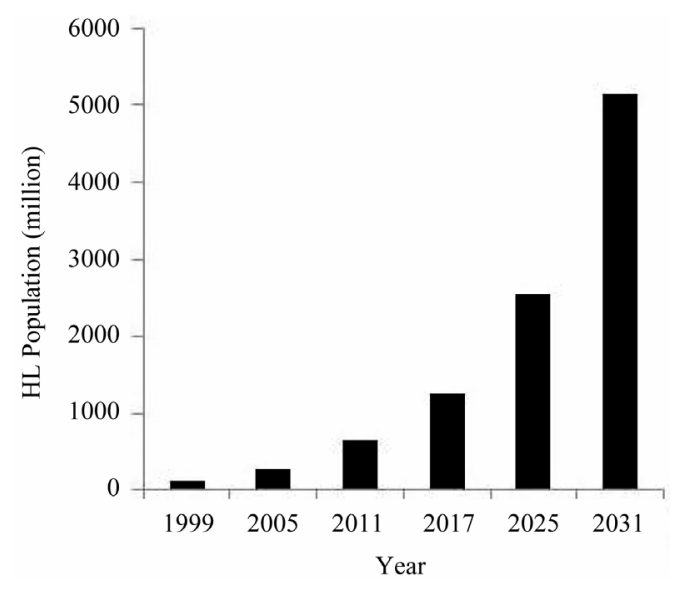

Figure 3. Prevalence of hearing loss as a function of year. The prevalence of hearing loss (HL) is presented as the total number of population who suffer from some sort of hearing impairment worldwide each year. The total numbers of people who suffer from some sort of hearing impairment increases with each year.

populations shown in Figure 3 which the World Health Organization found for the year from 1999 to 2011, we can observe a trend where the number has more than doubled every 6 years. Therefore, we propose that the population will exceed 1284 million in 2017 (i.e., 642 million $\times 2$ ), and will exceed 2568 million in 2025 (i.e., 1284 million $\times 2$ ).

It may be interesting to compare worldwide population at senior age with the worldwide hearing impaired population at all ages. This can be achieved for the year of 2025 as the values for both categories can be derived. Based on the number proposed by the United Nations Population Fund, by 2025, the number of people in the world aged 65 and over will reach around 1200 million [35]. Based on the number as we proposed above, by 2025, the number of hearing impaired people will reach 2568 million. Based on study of hearing impaired for the elderly people, the prevalence can reach $40 \%$ among the seniors at age of 65 or above (Figure 1). Therefore, the number of hearing impaired population in the elderly will be around 1200 million $\times 40 \%=480$ million.

The number can also be derived from total world population in 2025 (7937 million) [35] and percentage of seniors in the population in 2025 (17\%, interpolated from Figure 2). The seniors will be 1350 million $(7937 \times 17 \%)$ in 2025, and the seniors with hearing loss will be 540 million $(1350 \times 40 \%$, Figure 1). The numbers of either hearing impairment groups in all ages (2568 million) and in seniors (510 million, means of 480 and 540 ) is overwhelming, and so, the challenge to meet this ever growing demand is overwhelming as well.

However, we hope that via birth control, improvement of health services, and research on presbycusis, the number of hearing loss can reduce by $30 \%$ from 2568 to 1600 million in all ages and from 510 to 340 million in seniors.

\subsection{Hearing Impairment: Ranking the Third Place in Prevalence among All Chronic Health Conditions in Elderly Residents}

An estimate of the number of Canadians with hearing loss in assistive living facilities (or residential care facilities) is approximately 125,000 , and this number is about half of the total residents living in these care facilities $[8,9]$. The number can be even higher, for example, some estimates of the prevalence of hearing loss in seniors living in senior facilities in the United States are as high as $90 \%$ [9,36,37].

Although estimates can be different, which could be due to difference in criteria, the results can be alarming because hearing impairment ranks the third in prevalence among all chronic health conditions in elderly residents worldwide, which is superseded only by arthritis and hypertension [9-13].

\subsection{Hearing Loss in Elderly Neuroscience (I): A Negative Factor on Mental Health and Social Life}

Hearing loss in older persons affects their quality of life, their cognitive/emotional/social functions, and their roles in the family and nursing home [14]. The non-auditory effects of hearing loss such as mental health and quality of life in the elderly were highlighted by some investigators [38]. They concluded that sensory hearing loss tends to negatively affect the quality of life of the elderly, interfering with their capacity to communicate, and affecting mood and the level of participation in social life [38]. This may be independent of the cognitive and physical state of individuals. In the long term and in many cases the negativity may end in depression. Therefore, they recommended early detection and treatment of hearing loss as a crucial bio-psycho-social benefit to the elderly.

Investigators found that experiencing a decline in hearing was associated with increased distress [30]. The results they found were supported on the basis of statistical significance. This can be true as hearing loss presents as a negative effect on various aspects in the elderly.

Hearing loss can also contribute to the isolation behavior, depression, and possibly dementia as well [2,4, 39]. It was also linked to mild memory impairment [40].

The mood influence has been emphasized by Preminger et al. [41]. They found that in people with hearing loss and their significant partners and relatives, perceptions of hearing loss-related quality of life is highly correlated with negative mood scores [41]. They demonstrated that incongruence based on the scores of relation 
of hearing loss with quality of life, reported by members of a couple, was highly correlated with negative effect of mood measured within individuals.

Therefore, early detection and treatment of hearing loss to improve bio-psycho-social benefit to the elderly has been highly recommended [38].

\subsection{Hearing Loss in Elderly Neuroscience (II): A Negative Effect on Speech Perception and Hearing-Related Areas in the Brain}

Seniors may experience difficulty in an environment with multiple speakers [9,42]. Such deterioration in speech perception and cognition may be due to additional changes in the central auditory nervous system. Various studies indicate that deterioration of cognition is associated with multiple speech processing related components [15, 16]. For example, they include selective attention, working memory, long-term memory, speed of processing, inhibitory function, and executive functioning $[15,16]$. These deteriorations are significantly associated with agerelated reduction in brain structure, size and white matter integrity $[15,16]$.

Bernstein emphasized that the most important aspects of central auditory processing of speech are the auditory processing of interaural timing information [43]. GordonSalant attributed these problems specifically to the limited acoustic cues for temporal and binaural processing [44]. It was reported that elderly people were commonly challenged by three aspects of temporal distortions; time compression, noise, and reverberation in everyday listening situations. For example, the seniors frequently show poorer recognition of rapid speech or time-compressed speech than younger listeners [45]. GordonSalant clarified that this may be due to the limited acoustic cues for consonants that are associated with rapid speech [44]. These authors also found that the selective training in speech segments may improve recognition performance by elderly listeners.

Stach et al. also described central presbycusis in terms of age [46]. They found that central auditory processing problems were demonstrated in only $17 \%$ of their subjects who were less senior such as in 50 to 54 years old while in as high as $90 \%$ to $95 \%$ in their more senior subjects such as in $>80$ years old. Furthermore, Jerger et al. proposed a possibility that the higher prevalence of central presbycusis in older persons can be neither purely due to a reflection of hearing loss in threshold nor principally due to a cognitive change [47].

Statistic data in North America based on subjective responses to questionnaires reflect that more seniors experienced hearing handicap, regardless of the type or degree of hearing impairment. For example, using this same kinds of questionnaire items, in a survey on community-living seniors in a North American district in
1988 , it was found that $20 \%$ of people reported difficulty in one-to-one conversation and 33\% of them reported difficulty in conversation with two or more people $[9,42]$. Seniors who reported difficulties in group conversations, but not in dialogues, would be expected still to have good peripheral hearing (i.e., pure-tone thresholds) but possibly already have co-occurring central auditory or cognitive involvement.

Even though the changes in the inner ear can cause hearing loss, but such change may further affect the brain [48]. Dillon suggested that the phenomenon of decreased speech discrimination ability may be more complex than just merely a lack of stimulation (auditory inactivity or lazy ear) [48]. He further stated that the term deprivation is applicable since the underlying cause can be an inadequate output from the inner ear to the brain. Such deleterious consequences due to lack of output from inner ear to the brain can occur as the auditory pathways and areas in the brain are "starved" of sound, followed by atrophy which may occur after starvation of sound for several years $[49,50]$. Therefore, one of the causes of decreased speech discrimination may also be due to an inadequate output from the hearing organ to the brain, as agreed by [48].

Additionally, a recent study showed positive correlation between the decreased ability to perceive speech in noise in older adults and decline in the relative volume of the left ventral and dorsal prefrontal cortex [51]. This suggests that the central nervous system contributes to the ability to perceive speech in noise.

The concept of delayed auditory deprivation was also delineated $[49,50]$. For adults, a hearing loss can be typically left unattended for several years. This deprivation results in a situation where the auditory pathways and areas in the brain are "starved" of sound, and atrophy occurs.

Research has shown consistently that when there is a hearing loss in both ears and only one ear is fit with a hearing aid, the auditory nerve in the unaided ear can atrophy, resulting in auditory deprivation, defined as a gradual decline in auditory function due to reduction of the acoustic information. This asymmetrical setup causes one ear to take on more of the listening activity than the other. This results in the weakening of the unaided ear over time [48]. If hearing loss only occurs in one ear without treatment or occurs in both ears but only with one ear being treated, atrophy can occur in the affected side or untreated side. The patient loses the auditory balance between the two ears.

\subsection{Clinical Significance: Challenge in Management and Prevention of the Presbycusis}

By 2025, there will be an estimated around 1200 million 
people in the world aged 65 and over [35]. The percentage of the population aged 65 years and older is expected to rise to $21.8 \%$ by 2031 [8,35]. If $40 \%$ of the population aged 65 years or older is hearing impaired [18,31], the number of hearing impaired in the elderly population will be overwhelming, which will cast a tremendous challenge on how to manage ever increasing cases of presbycusis.

Hearing rehabilitation for seniors may not be simple because they may have additional central auditory processing disorders [52]. Such central auditory processing disorders among the elderly have been reported as $10 \%$ $20 \%$ in the general population [53], but $80 \%-90 \%$ in the clinical population [46,53].

Levy et al. (2006) assumed that age stereotypes could contribute to the adverse health behavior consequences [54]. In the absence of a routine medical intervention for hearing loss, the possibility of psychosocial explanation takes on additional importance [19]. Fozard et al. concluded that older individuals, in contrast to younger individuals, are more accepting of hearing loss and less likely to seek professional care [55]. On the other hand, seniors may experience difficulty benefiting from and accessing existing services which may be adequate for the younger adult [35].

As demonstrated above that age-related hearing loss is associated with cognitive decline, to facilitate optimal functional capacity in patients and perform a more comprehensive management is needed. The assessment should include measures of central auditory function such as dichotic tasks and speech-in-noise testing. Treatment should include auditory rehabilitative training and counseling [56].

As hearing loss in seniors is usually more severe and more complex than in the younger population, better hearing aids are often desired. Among consumers switching to newly-developed hearing devices, the average age for such switching has increased to nearly 70 years old [57]. This indicates that at age 70 the seniors feel difficult to wait for a better device. Switching to a better device or a better program installed in the device typically occurs every 2 years in the elderly [58].

Humes and colleagues evaluated the usage of hearing aids by using several independent dimensions of hearing aid outcomes including subjective benefit, aided speech recognition performance, objective benefit, satisfaction, and usage [58-60]. They found that although hearing aid usage and satisfaction declines over a longer term, measures of hearing aid satisfaction in a shorter term such as 1 month and 6 months post-fitting were generally stable. Such results could be maintained for up to 2 years after the delivery of the hearing aids [58-61]. This 2-year period matched the 2-year period to switch to a better device as mentioned above [58]. Although not confirmed, advanced fitting such as real-ear-to-coupler approach may extend the period of satisfaction [62].

Income may be associated with access to medical services, and higher minimum wage rates were associated with reduced odds of reporting unmet medical needs [63]. Therefore, budget may become another obstacle. Compared to pre-retirement, the retired elderly have lower income and limited insurance coverage. In addition, the price of co-pay for hearing device increased in estimation by $7.3 \%$ in average in the period from 1984 to 2004 but by $14.6 \%$ for the higher price behind-ear model (BTE) and by $19.1 \%$ for the higher price in-the-ear model (ITE). Therefore, hearing aid cost is another obstacle that hinders elderly from having the proper rehabilitation. [30] thought that further investigation into the factors associated with hearing aid use would be necessary to better target older adults who might benefit from wearing a hearing aid.

Cochlear implants have given a great hope [64]. Cochlear implant users show a twofold improvement in overall quality of life scores when compared to hearing aid users in the severe and profound hearing loss spectrum [64]. However, not all subjects who need cochlear implants may obtain an opportunity being implanted due to various reasons. The candidacy issue may also restrict the elderly receiving the implant although this issue has not been raised for the elderly [65].

Although presbycusis is hard to prevent as it is due to degenerative changes, prevention of confounding factors can be an important factor in reducing the deterioration of the presbycusis condition because the confounding factors can magnify the hearing loss consequences. For example, ototoxic drugs (Antibiotics such as aminoglycosides or chemotherapeutic agents) can be a confounding factor [66].

In addition, noise that is even at moderate intensity can accelerate the presbycusis process [67]. The ear protector may be used as a protection against noise exposure as using ear protectors can reduce the noise by $15 \mathrm{~dB}$ [68]. Such protection can be effective in preventing further deterioration of hearing. In addition to prevention and early treatment of other confounding medical conditions such as heart diseases, hyperlipidemia and diabetes can be essential to the protection against further hearing damage [69].

\section{Conclusions}

Presbycusis is a critical issue in our community, which is summarized below:

- Population worldwide increases yearly, the number of people with hearing loss increases yearly, the percentage of seniors in society increases yearly, the prevalence of presbycusis increases with years of age, and therefore the number of seniors with hearing loss 
increases yearly in an alarming speed in the world;

- Projected world population is 7937 million in 2025;

- Estimated $14.5 \%$ of population is over age 65 by $2011,21.8 \%$ by 2031, and interpolated $17 \%$ by 2025 ;

- Hearing loss affected 642 million people worldwide in 2011 and 2568 millions in 2025 in estimation;

- Older people are subject to more hearing loss. Based on the values of 7937 million and 17\% above, people over 65 are estimated around 1350 million by 2025;

- The prevalence of hearing loss can be up to $40 \%$ of the seniors above age 65 and $80 \%$ of them above 85 ;

- The values above (7937, 2568 and 1350 million as well as $17 \%$ and $40 \%$ ) project that hearing loss may affect up to 540 million seniors by 2025 ;

- Hearing impairment ranks the 3rd place in prevalence among all chronic health conditions in elderly residents worldwide, which is superseded only by arthritis and hypertension;

- Sensory, strial, and neural degenerations are the main etiology in presbycusis besides hereditary susceptibility and change in the central nervous system;

- Hearing loss is also a negative factor on the mental health, social wellbeing, speech perception, and hearing-related areas in the brain in the elderly;

- Therefore, hearing rehabilitation for seniors may not be simple due to additional central auditory processing disorders often seen in the clinical population;

- Based on the critical situations as described above, the clinics will face a significant challenge in management of presbycusis such as a foreseen heavy case load, need for considering mental involvement, more complexity in applying assistive devices;

- Although prevention is hard due to the degenerative nature of the presbycusis, prevention of confounding factors can be important such as ototoxic drugs and deafen noise. In addition, confounding factors can include heart diseases, hyperlipidemia, and diabetes.

\section{Acknowledgements}

This work was supported by Research Funding from the University of Alberta to MZ. We thank Pat Jones for effective editing and proofreading, Dr. Melanie Campbell and Brian Schmidt for insightful discussions of the manuscript, and anonymous reviewers for their very valuable comments.

\section{REFERENCES}

[1] P. S. Ronald, A. D. Meyers, et al., "Presbycusis,” 2013. http://emedicine.medscape.com/article/855989-overview

[2] G. A. Gates and J. H. Mills, "Presbycusis," Lancet, Vol. 366, No. 9491, 2005, pp. 1111-1120. doi:10.1016/S0140-6736(05)67423-5

[3] K. Woodcock and J. D. Pole, "Educational Attainment,
Labour Force Status and Injury: A Comparison of Canadians with and without Deafness and Hearing Loss," International Journal of Rehabilitation Research, Vol. 31, No. 4, 2008, pp. 297-304.

doi:10.1097/MRR.0b013e3282fb7d4d

[4] L. M. Corna, et al., "Transitions in Hearing Impairment and Psychological Distress in Older Adults,” Canadian Journal of Psychiatry, Vol. 54, No. 8, 2009, pp. 518-525.

[5] H. F. Schuknecht and M. R. Gacek, "Cochlear Pathology in Presbycusis," Annals of Otology, Rhinology, and Laryngology, Vol. 102, No. 1, 1993, pp. 1-16.

[6] M. I. Wallhagen, et al., “An Increasing Prevalence of Hearing Impairment and Associated Risk Factors over Three Decades of the Alameda County Study,” American Journal of Public Health, Vol. 87, No. 3, 1997, pp. 440442. doi:10.2105/AJPH.87.3.440

[7] Ministry of Supply and Services Canada, "Ageing and Independence: Overview of a National Survey. Seniors Secretariat, Health and Welfare Canada, Ottawa, on Catalogue h88-3/13-1993e,” 1993.

[8] Statistics Canada, "Canadians with Impaired Hearing, Special Topic Series," The Health and Activity Limitation Survey \#5 of 6 . Catalogue 82-615, Ottawa, Statistics Canada, 1992.

[9] K. Pichora-Fuller and M. Cheesman, "Preface to the Special Issue on Hearing and Aging," Journal of SpeechLanguage Pathology and Audiology, Vol. 21, No. 2, 1997, pp. 75-79.

[10] C. A. Binnie, "The Future of Audiological Rehabilitation: Overview and Forecast," Journal of the Academy of Rehabilitative Audiology Monographs, Vol. 27, 1994, pp. 13-24.

[11] D. Haber, "Health Promotion and Aging: Practical Applications for Health Professionals,” 4th Edition, Springer Publishing Company, New York, 1994.

[12] M. E. Lutman, "Hearing Disability in the Elderly," Acta Oto-Laryngologica. Supplementum, Vol. 476, 1990, pp. 239-248.

[13] K. J. Cruickshanks, et al., "Prevalence of Hearing Loss in Older Adults in Beaver Dam, Wisconsin. The Epidemiology of Hearing Loss Study," American Journal of Epidemiology, Vol. 148, No. 9, 1998, pp. 879-886. doi:10.1093/oxfordjournals.aje.a009713

[14] J. Katz, "Handbook of Clinical Audiology,” Lippincott Williams \& Wilkins, Baltimore, 2002.

[15] M. Isingrini, A. Perrotin and C. Souchay, "Aging, Metamemory Regulation and Executive Functioning," Progress in Brain Research, Vol. 169, 2008, pp. 377-392.

[16] D. C. Park and P. Reuter-Lorenz, "The Adaptive Brain: Aging and Neurocognitive Scaffolding," Annual Review of Psychology, Vol. 60, 2009, pp. 173-196.

[17] K. J. Rothman, "Epidemiology: An Introduction,” 2nd Edition, Oxford University Press, Oxford, 2012.

[18] B. Yueh, et al., "Screening and Management of Adult Hearing Loss in Primary Care: Scientific Review," JAMA, Vol. 289, No. 15, 2003, pp. 1976-1985. doi:10.1001/jama.289.15.1976

[19] S. T. Bogardus Jr., B. Yueh and P. G. Shekelle, “Screen- 
ing and Management of Adult Hearing Loss in Primary Care: Clinical Applications,” JAMA, Vol. 289, No. 15, 2003, pp. 1986-1990. doi:10.1001/jama.289.15.1986

[20] National Center for Health Statistics, "New Series of Reports to Monitor Health of Older Americans: Trends in Vision and Hearing among Older Americans," Aging Trends, Vol. 2, 2001, pp. 1-9.

[21] N. McGauran, et al., "Reporting Bias in Medical Research-A Narrative Review," Trials, Vol. 11, 2010, p. 37.

[22] J. A. Collins and B. C. Fauser, "Balancing the Strengths of Systematic and Narrative Reviews," Human Reproduction Update, Vol. 11, No. 2, 2005, pp. 103-104. doi:10.1093/humupd/dmh058

[23] H. Zwaardemaker, "Der Verlust an Hornen Tonen Mit Zunehemendum Alter: Ein Neues Gesetz,” Arch Ohr NasKehlk-Heilk, Vol. 32, 1891, p. 53.

[24] J. H. Mills, R. A. Schmidt and J. R. Dubno, “Age-Related Hearing Loss: A Loss of Voltage Not Hair Cells,” Semin Hear, Vol. 27, No. 5, 2006, pp. 228-236. doi:10.1055/s-2006-954849

[25] J. Rodriguez-Paris, et al., "Genetic Analysis of Presbycusis by Arrayed Primer Extension," Annals of Clinical \& Laboratory Science, Vol. 38, No. 4, 2008, pp. 352-360.

[26] M. Unal, et al., "N-Acetyltransferase 2 Gene Polymorphism and Presbycusis,” Laryngoscope, Vol. 115, No. 12, 2005, pp. 2238-2241. doi:10.1097/01.mlg.0000183694.10583.12

[27] E. G. Nelson and R. Hinojosa, "Presbycusis: A Human Temporal Bone Study of Individuals with Downward Sloping Audiometric Patterns of Hearing Loss and Review of the Literature," Laryngoscope, Vol. 116, No. 9, 2006, pp. 1-12.

[28] M. Zhang and J. G. Surles, "Voltage-Dependent Outer Hair Cell Stereocilia Stiffness at Acoustic Frequencies,” Neuroreport, Vol. 19, No. 8, 2008, pp. 855-859. doi:10.1097/WNR.0b013e3282ff84e8

[29] A. F. Ryan, "Protection of Auditory Receptors and Neurons: Evidence for Interactive Damage," Proceedings of the National Academy of Sciences of the United States of America, Vol. 97, No. 13, 2000, pp. 6939-6940. doi:10.1073/pnas.97.13.6939

[30] L. M. Corna, et al., "Corrected and Uncorrected Hearing Impairment in Older Canadians,” Gerontology, Vol. 55, No. 4, 2009, pp. 468-476. doi:10.1159/000219589

[31] R. D. Frisina and J. P. Walton, "Age-Related Structural and Functional Changes in the Cochlear Nucleus," Hearing Research, Vol. 216-217, 2006, pp. 216-223.

[32] J. F. Willott, "Aging and the Auditory System: Anatomy, Physiology, and Psychophysics,” Singular Pub Group, San Diego, 1991.

[33] Fact-Sheet, "Canadian Association of Speech-Language Pathologists and Audiologists,” 2013. http://www.speechandhearing.ca/files/caslpa_fact_sheet.p df

[34] P. Raina, et al., "Prevalence, Risk Factors and Self-Reported Medical Causes of Seeing and Hearing-Related Disabilities among Older Adults," Canadian Journal on
Aging, Vol. 19, No. 2, 2000, pp. 260-278. doi:10.1017/S0714980800014045

[35] UNFPA and HelpAge International, "Ageing in the Twenty-First Century,” UNFPA and HelpAge International, New York, 2012.

[36] R. Hull and K. M. Griffin, "Communication Disorders in Aging,” Sage Publications, Newbury Park, 1989.

[37] R. L. Schow and M. A. Nerbonne, "Hearing Levels among Elderly Nursing Home Residents," The Journal of Speech and Hearing Disorders, Vol. 45, No. 1, 1980, pp. 124-132.

[38] J. C. Millan-Calenti, et al., "Relationship between Sensory Hearing Loss and Depression in Elderly People: A Literature Review," Revista Española de Geriatría y Gerontología, Vol. 46, No. 1, 2011, pp. 30-35.

[39] K. Woodcock and J. D. Pole, "Health Profile of Deaf Canadians: Analysis of the Canada Community Health Survey," Canadian Family Physician, Vol. 53, No. 12, 2007, pp. 2140-2141.

[40] G. A. Gates, et al., "Central Auditory Dysfunction in Older Persons with Memory Impairment or Alzheimer Dementia," Archives of Otolaryngology-Head \& Neck Surgery, Vol. 134, No. 7, 2008, pp. 771-777. doi:10.1001/archotol.134.7.771

[41] J. E. Preminger and S. Meeks, "The Influence of Mood on the Perception of Hearing-Loss Related Quality of Life in People with Hearing Loss and Their Significant Others," International Journal of Audiology, Vol. 49, No. 4, 2010, pp. 263-271. doi:10.3109/14992020903311396

[42] Regional Report, "Regional Municipality of HamiltonWentworth and Hamilton-Wentworth District Health Council. Services of Seniors Study-Mapping the Way to the Future for the Elderly: Report of Findings and Recommendations,” 1988.

[43] L. R. Bernstein, "Auditory Processing of Interaural Timing Information: New Insights,” Journal of Neuroscience Research, Vol. 66, No. 6, 2001, pp. 1035-1046. doi:10.1002/jnr.10103

[44] S. Gordon-Salant and P. J. Fitzgibbons, "Sources of AgeRelated Recognition Difficulty for Time-Compressed Speech," Journal of Speech, Language, and Hearing Research, Vol. 44, No. 4, 2001, pp. 709-719. doi:10.1044/1092-4388(2001/056)

[45] K. S. Helfer and L. A. Wilber, "Hearing Loss, Aging, and Speech Perception in Reverberation and Noise," Journal of Speech and Hearing Research, Vol. 33, No. 1, 1990, pp. 149-155.

[46] B. A. Stach, M. L. Spretnjak and J. Jerger, "The Prevalence of Central Presbyacusis in a Clinical Population," Journal of the American Academy of Audiology, Vol. 1, No. 2, 1990, pp. 109-115.

[47] J. Jerger, et al., "Speech Understanding in the Elderly," Ear and Hearing, Vol. 10, No. 2, 1989, pp. 79-89. doi:10.1097/00003446-198904000-00001

[48] H. Dillon, "Hearing Aids," Thieme Boomerang Press, Sydney, 2001.

[49] S. Silman, et al., "Prediction of Hearing Loss from the Acoustic-Reflex Threshold,” In: S. Silman, Ed., The Acous- 
tic Reflex: Basic Principles and Clinical Applications, Academic Press, New York, 1984, pp. 187-203. doi:10.1016/B978-0-12-643450-7.50011-1

[50] S. Silman, et al., “Adult-Onset Auditory Deprivation,” Journal of the American Academy of Audiology, Vol. 3, No. 6, 1992, pp. 390-396.

[51] P. C. Wong, et al., "Neuroanatomical Characteristics and Speech Perception in Noise in Older Adults," Ear and Hearing, Vol. 31, No. 4, pp. 471-479. doi:10.1097/AUD.0b013e3181d709c2

[52] R. Chmiel and J. Jerger, "Hearing Aid Use, Central Auditory Disorder, and Hearing Handicap in Elderly Persons," Journal of the American Academy of Audiology, Vol. 7, No. 3, 1996, pp. 190-202.

[53] J. C. Cooper Jr. and G. A. Gates, "Hearing in the Elderly-The Framingham Cohort, 1983-1985: Part II. Prevalence of Central Auditory Processing Disorders," Ear and Hearing, Vol. 12, No. 5, 1991, pp. 304-311. doi:10.1097/00003446-199110000-00002

[54] B. R. Levy, M. D. Slade and T. M. Gill, "Hearing Decline Predicted by Elders' Stereotypes," The Journals of Gerontology: Series B, Vol. 61, No. 2, 2006, pp. P82-P87. doi:10.1093/geronb/61.2.P82

[55] J. L. Fozard and S. Gordon-Salant, "Changes in Vision and Hearing with Aging," In: J. E. Birren and K. W. Schaie, Eds., Handbook of Psychology of Aging, Academic Press, New York, 2001, pp. 214-266.

[56] K. Parham, et al., "Comprehensive Management of Presbycusis: Central and Peripheral,” Otolaryngology—Head and Neck Surgery, Vol. 148, No. 4, 2013, pp. 537-539. doi:10.1177/0194599813477596

[57] S. Kochkin, "MarkeTrak VII: Hearing Loss Population Tops 31 Million People. The Hearing-Impaired Population Continue to Increase-Along with Satisfaction Rating of Hearing Instruments,” Hearing Review, Vol. 12, No. 7, 2005, pp. 16-29.

[58] L. E. Humes, et al., "Longitudinal Changes in Hearing Aid Satisfaction and Usage in the Elderly over a Period of One or Two Years after Hearing Aid Delivery,” Ear and Hearing, Vol. 23, No. 5, 2002, pp. 428-438. doi:10.1097/00003446-200210000-00005

[59] L. E. Humes, "Dimensions of Hearing Aid Outcome," Journal of the American Academy of Audiology, Vol. 10, No. 1, 1999, pp. 26-39.

[60] L. E. Humes, "Issues in Evaluating the Effectiveness of
Hearing Aids in the Elderly: What to Measure and When," Seminars in Hearing, Vol. 22, No. 3, 2001, pp. 303-314. doi:10.1055/s-2001-15633

[61] L. E. Humes, et al., "Hearing-Aid Outcome Measured Following One Month of Hearing Aid Use by the Elderly," Journal of Speech, Language, and Hearing Research, Vol. 44, No. 3, 2001, pp. 469-486. doi:10.1044/1092-4388(2001/037)

[62] S. Scollie, et al., "Accuracy and Reliability of a Real-Earto-Coupler Difference Measurement Procedure Implemented within a Behind-the-Ear Hearing Aid,” Journal of the American Academy of Audiology, Vol. 22, No. 9, 2011, pp. 612-622. doi:10.3766/jaaa.22.9.6

[63] K. P. McCarrier, et al., "Associations between Minimum Wage Policy and Access to Health Care: Evidence from the Behavioral Risk Factor Surveillance System, 19962007,” American Journal of Public Health, Vol. 101, No. 2, 2011, pp. 359-367. doi:10.2105/AJPH.2006.108928

[64] S. M. Cohen, et al., "Quality of Life in Hearing-Impaired Adults: The Role of Cochlear Implants and Hearing Aids," Otolaryngology_Head and Neck Surgery, Vol. 131, No. 4, 2004, pp. 413-422. doi:10.1016/j.otohns.2004.03.026

[65] E. Fitzpatrick, et al., "Pediatric Cochlear Implantation: How Much Hearing Is Too Much?” International Journal of Audiology, Vol. 48, No. 2, 2009, pp. 91-97. doi:10.1080/14992020802516541

[66] B. A. Prieve and J. L. Yanz, "Age-Dependent Changes in Susceptibility to Ototoxic Hearing Loss," Acta Oto-Laryngologica, Vol. 98, No. 5-6, 1984, pp. 428-438. doi:10.3109/00016488409107584

[67] P. Campo, et al., "Impact of Noise or Styrene Exposure on the Kinetics of Presbycusis," Hearing Research, Vol. 280, No. 1-2, 2011, pp. 122-132. doi:10.1016/j.heares.2011.04.016

[68] K. H. Huttunen, V. P. Sivonen and V. T. Poykko, "Symphony Orchestra Musicians' Use of Hearing Protection and Attenuation of Custom-Made Hearing Protectors as Measured with Two Different Real-Ear Attenuation at Threshold Methods,” Noise Health, Vol. 13, No. 51, 2011, pp. 176-188. doi:10.4103/1463-1741.77210

[69] C. S. Sousa, et al., "Risk Factors for Presbycusis in a Socio-Economic Middle-Class Sample,” Brazilian Journal of Otorhinolaryngology, Vol. 75, No. 4, 2009, pp. 530-536. doi:10.1590/S1808-86942009000400011 